\title{
Fermion production during preheating after hybrid inflation
}

\author{
Juan García-Bellido \\ Departamento de Física Teórica, C-XI, Universidad Autónoma de Madrid \\ 28049 Madrid, Spain \\ Email: bellido@delta.ft.uam.es'
}

\section{Silvia Mollerach and Esteban Roulet}

Departamento de Física, Universidad Nacional de La Plata

CC 67, 1900, La Plata, Argentina

Email: molieracevenus. fisica.unlp.edu. ari,

rouletevenus. fisica.unlp.edu. ar

Abstract: At the end of inflation, the coherent oscillations of the inflaton field may resonantly amplify the long wavelength modes of both bosons and fermions coupled to it. We study the resonant production of both kinds of particles during preheating in a model of hybrid inflation. The coherent time evolution of the inflaton and the Higgs fields after inflation induce a very different production of fermions depending on whether they are coupled to the Higgs or to the inflaton. For reasonable values of the model parameters, the fermion production through parametric resonance can be very efficient. We study the relative growth of the fermion and boson energy densities during preheating in hybrid models. During the initial stage of preheating, fermion production dominates the relative energy density, while the exponential growth of bosonic modes soon takes over.

KEYwords: 'Cosmology of Theories beyond the SM, Physics of the Early Universè. 


\section{Contents}

introduction Intricis

2. Hybrid model

3. Pair production of fermions

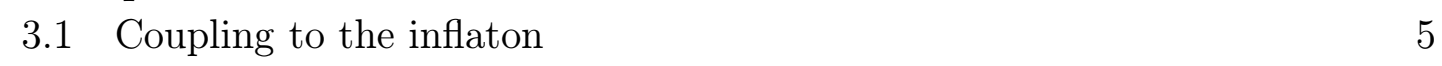

13.2. Coupling to the Higgs

这. Boson production

75. Conclusions

\section{Introduction}

Cosmological inflation is an extremely efficient mechanism in diluting any particle species or fluctuations. At the end of inflation, the universe is empty and extremely cold, dominated by the homogeneous coherent mode of the inflaton. Its potential energy density is converted into particles, as the inflaton field oscillates around the minimum of its potential. These particles are initially very far from equilibrium, but they interact among themselves (or decay) and thermal equilibrium is achieved at a very large temperature. From there on the universe expanded isoentropically, cooling down as it expanded, in the way described by the standard hot Big Bang model. Recent developments in the theory of reheating suggest that the decay of the inflaton energy could have been explosive [i] $\left[\begin{array}{l}1 \\ 1\end{array}\right]$, due to the coherent oscillations of the inflaton zero mode, which induce its stimulated decay. The result is a resonant production of particles in just a few inflaton oscillations, in a process known as preheating [i]. The number of particles produced in this way is exponentially large, which may account for the extraordinarily large entropy, of order $10^{89}$, in our observable patch of the universe today.

Preheating is not generic, it may occur in different models of inflation [2, i but only under special circumstances. Preheating strongly depends on the inflaton couplings to other fields, as well as on the amplitude and frequency of the inflaton oscillations. This explosive production occurs through parametric resonance of the long wavelength modes of any field coupled to the inflaton, either bosonic in or fermionic [5. Due to the parametric nature of the resonance the particle production 
occurs for well defined frequency bands. The difference between bosonic or fermionic preheating is spectral: bosons can have large occupation numbers for a single mode, while fermions saturate due of the Pauli exclusion principle, and therefore a larger fraction of their energy can be transferred to higher momentum modes. Also, the rate of growth of these two components is very different: while the boson energy

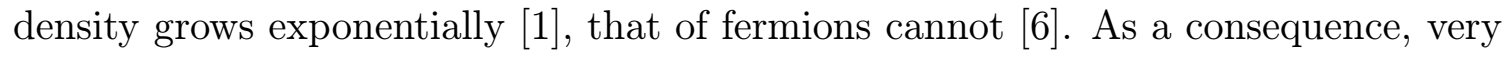
soon bosons dominate the decay of the inflaton energy if both kinds of particles are resonantly produced. After a few inflaton oscillations, the energy density in fermions and bosons may be large enough to backreact on the inflaton oscillations and hence this will eventually stop their resonant production $\left[\begin{array}{l}\overline{2} \\ \underline{2}\end{array}, \overline{\bar{i}}\right]$.

After preheating in chaotic inflation, the main part of the energy density of the universe is in the coherent modes of bosons: the inflaton, as well as any other parametrically amplified bosonic fields with large occupation numbers, while a subdominant part may be in the parametrically produced fermions. This state is very far from thermal equilibrium (characterized by a decoherent ensemble with small occupation numbers for all momentum modes) and, in fact, such a state could be used to generate the required baryon asymmetry during preheating, either via GUT baryogenesis [8] $\left[\begin{array}{l}8 \\ 0\end{array}\right.$ of the universe occurs through the rescattering of all the particles, which breaks the coherence of the bosonic fields. See, for instance, ref. [1] $\left.\overline{0}_{1}^{]}\right]$for thermalization via fermionic modes.

In this paper we study the resonant production of both fermions and bosons in a hybrid model of inflation [i $\left[\begin{array}{l}1 \\ 1\end{array}\right]$. In these models it is possible to choose, without finetuning, the masses and couplings of the fields in such a way that the rate of expansion is negligible compared to the masses involved [i] in a few inflaton oscillations after the end of inflation, before the scale factor has grown by a single e-fold, and therefore we can ignore the universe expansion. This reduces the problem to resonant production of fermions in Minkowski space, for which there is a complete analytic treatment [6] $[6]$. We find that in the case of hybrid inflation, contrary to what happens in chaotic inflation, it is possible to produce, for certain couplings of the fermions to the inflaton field, a larger contribution of fermionic modes than bosonic ones to the energy density of the universe at the end of preheating.

We describe in section $i_{-1}^{15}$ the hybrid model under consideration, with a Higgstype field coupled to the inflaton, and discuss the solution of the classical equations of motion. In section ' $\overline{3}_{1}$, we study the pair production of fermions in this model, for a non-zero coupling of fermions to both the inflaton and the Higgs field. We analyze the growth of the fermion energy density for different values of the couplings, as well as for different fermion masses. In section ${ }_{-1}^{\prime}$ we study the boson production for both couplings to inflaton and Higgs, together with the growth of the bosonic energy density. In section ${ }_{-1}^{\prime 2}$, we draw our conclusions. 


\section{Hybrid model}

We will consider a model of inflation with two fields, one that slow-rolls down a potential, driving inflation, and another one with a symmetry breaking potential that triggers the end of inflation, which we will call the Higgs. Such a model is called hybrid inflation and was proposed by Linde long ago [1] $\left.\overline{1}_{1}^{1}\right]$. There are many particle physics realizations of this general class of models potential for hybrid inflation is given by the tree level potential ${ }^{1}$

$$
V(\phi, \sigma)=\frac{M^{4}}{4 \lambda}-\frac{1}{2} M^{2} \phi^{2}+\frac{1}{4} \lambda \phi^{4}+\frac{1}{2} g^{2} \phi^{2} \sigma^{2}+\frac{1}{2} m^{2} \sigma^{2}
$$

During hybrid inflation, the inflaton field $\sigma$ evolves along the Higgs valley at $\phi=0$. As soon as the Higgs acquires a negative mass term, it triggers the end of inflation. That is, in less than one e-fold the two fields start oscillating around their common absolute minimum $(\phi=v, \sigma=0)$, where $v=M / \sqrt{\lambda}$ is the Higgs vacuum expectation value (vev). The end of inflation occurs when the mass of the Higgs vanishes, i.e. at $\sigma=\sigma_{c} \equiv M / g$.

By defining $y \equiv \sigma / \sigma_{c}$ and $x \equiv \phi / v$, we can write the potential ( $\left(2 . \overline{1}{ }_{1}^{\prime}\right)$ as

$$
V(x, y)=V_{0}\left[\left(1-x^{2}\right)^{2}+2 x^{2} y^{2}+2 \gamma y^{2}\right]
$$

where $V_{0}=M^{4} / 4 \lambda$, and $\gamma=\lambda m^{2} / g^{2} M^{2}$ is a constant that is constrained to be small by the amplitude of the microwave background (CMB) anisotropies. It does not play any significant role after inflation and we will neglect it here. Also, the rate of expansion can be made relatively small in hybrid inflation (as long as $M \ll g M_{\mathrm{P}}$ ) and still satisfy the CMB constraints, see ref. [1] $1 \overline{2}]$. Therefore, we will ignore the rate of expansion of the universe here. We will also concentrate in the regime $\lambda \gg g^{2}$, for which the Higgs evolves along the minimum of the potential, following the inflaton oscillations.

We can then write the evolution equations after inflation, redefining the time unit as $\tau \equiv \bar{M} t$ (with $\bar{M}=g M / \sqrt{\lambda})$, as:

$$
\begin{aligned}
y^{\prime \prime}+\left(1-y^{2}\right) y & =0, \\
1-y^{2} & =x^{2} .
\end{aligned}
$$

For initial conditions $y(0)=y_{0}$ and $y^{\prime}(0)=0$, we find the solution

$$
\begin{array}{clrl}
y=y_{0} \operatorname{cd}(u \mid m), & \\
u=\tau \sqrt{\frac{a_{2}}{2}}, & m=\frac{a_{1}}{a_{2}}, \\
a_{1}=y_{0}^{2}, & a_{2}=2-y_{0}^{2},
\end{array}
$$

\footnotetext{
${ }^{1}$ See ref. [1] $\left.{ }_{1}^{\prime}{ }_{1}\right]$ for a discussion of loop-corrections to this potential during inflation.
} 


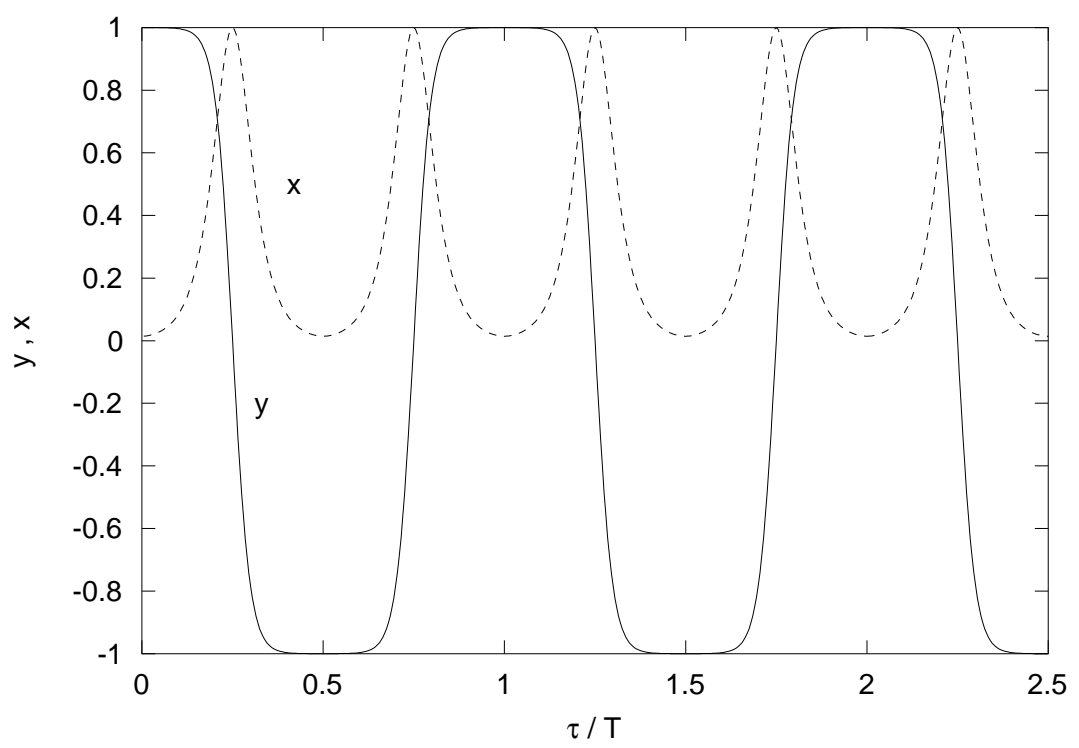

Figure 1: The time evolution of the inflaton and Higgs fields after inflation, for initial condition $y_{0}=0.9999$.

where $\operatorname{cd}(u \mid m)$ is the Jacobi elliptic function. This solution is periodic in the $\tau$ variable, with period

$$
T=4 \sqrt{\frac{2}{a_{2}}} K(m),
$$

with $K$ the elliptic integral. We have plotted the evolution of both the normalized Higgs and the inflaton after inflation in figure $y_{0}=0.9999$. The corresponding period of the inflaton oscillations is $T \simeq 29.97$. Although this period increases with $y_{0}$ approaching one, the mean energy density transferred to fermions coupled to the inflaton is nearly unchanged (provided that $y_{0}$ is close to one). If the fermions are coupled to the Higgs, the results are more sensitive to the value of $y_{0}$ for strong coupling, as we will discuss later.

\section{Pair production of fermions}

The oscillations of the inflaton and Higgs fields at the end of inflation trigger the explosive production of particles that couple to either or both of these fields. The production of bosons in hybrid inflation has been studied in ref. [3י-1, inj. Here we will analyze the fermion production in this model and in the next section will elaborate further on the bosonic case in order to compare the two results.

We will consider first the coupling of fermions to the inflaton $\sigma$, with coupling $h_{1} \sigma \bar{\psi} \psi$, and a possible mass term $m_{\psi} \bar{\psi} \psi$. Then we will consider the coupling of fermions to the Higgs, with coupling $h_{2} \phi \bar{\psi} \psi$. When the symmetry gets broken this last coupling will give the fermions a mass through the non vanishing vev of the Higgs. 


\subsection{Coupling to the inflaton}

Let us consider here a fermionic field $\psi$ satisfying the Dirac equation

$$
\left(i \gamma^{\mu} \nabla_{\mu}-h_{1} \sigma(t)-m_{\psi}\right) \psi=0
$$

The solutions are more easily obtained using an auxiliary field $X(\vec{x}, t)$, such that $\psi=\left(i \gamma^{\mu} \nabla_{\mu}+h_{1} \sigma(t)+m_{\psi}\right) X$. Decomposing it as $\exp (i \vec{K} \cdot \vec{x}) X_{K}(t) R_{r}$, with $R_{r}$ eigenvectors of $\gamma^{0}$ with eigenvalue +1 , we can write the equation of motion for fermion modes $X_{k}$ as [5]

$$
X_{k}^{\prime \prime}+\Omega_{k}^{2} X_{k}-i \sqrt{q_{1}} y^{\prime} X_{k}=0
$$

where

$$
\begin{aligned}
\Omega_{k}^{2}(\tau) & =k^{2}+\left(\sqrt{q_{1}} y(\tau)+\bar{m}_{\psi}\right)^{2}, \\
q_{1} & \equiv \frac{\lambda h_{1}^{2}}{g^{4}} \\
\bar{m}_{\psi} & \equiv \frac{m_{\psi}}{\bar{M}}
\end{aligned}
$$

and we have rescaled $k \equiv K / \bar{M}$. We will here display the results for $q_{1}$ between 1 and $10^{6}$. Notice that if we were to take e.g. $\lambda=1$ and $g=0.01$ this will lead to $q_{1}=10^{8} h_{1}^{2}$, and hence the values of $q_{1}$ considered would correspond to $h_{1}$ between $10^{-4}$ and 0.1 .

We take the initial conditions corresponding to positive frequency plane waves at $\tau<0$ :

$$
\begin{aligned}
& X_{k}(0)=\left[2 \Omega_{k}\left(\Omega_{k}+\sqrt{q_{1}} y_{0}+\bar{m}_{\psi}\right)\right]^{-1 / 2}, \\
& X_{k}^{\prime}(0)=-i \Omega_{k} X_{k}(0)
\end{aligned}
$$

The quantity of interest to us is the fraction of the total energy, $\rho_{\text {total }}=V_{0}$, which is transferred into fermions,

$$
\frac{\rho_{F}(\tau)}{\rho_{\text {total }}}=h_{1}^{2} \frac{2 N}{\pi^{2} q_{1}} \int d k k^{2} \Omega_{k}(\tau) n_{k}(\tau)
$$

with the number of fermion-pair degrees of freedom $N=1$ or 2 for Majorana or Dirac fields, respectively. The occupation number (for fermion pairs), $n_{k}$, can be calculated as [i] $\overline{1}$ in

$$
n_{k}(\tau)=\frac{1}{2}-\frac{k^{2}}{\Omega_{k}} \operatorname{Im}\left[X_{k} X_{k}^{\prime *}\right]-\frac{\sqrt{q_{1}} y+\bar{m}_{\psi}}{2 \Omega_{k}} .
$$




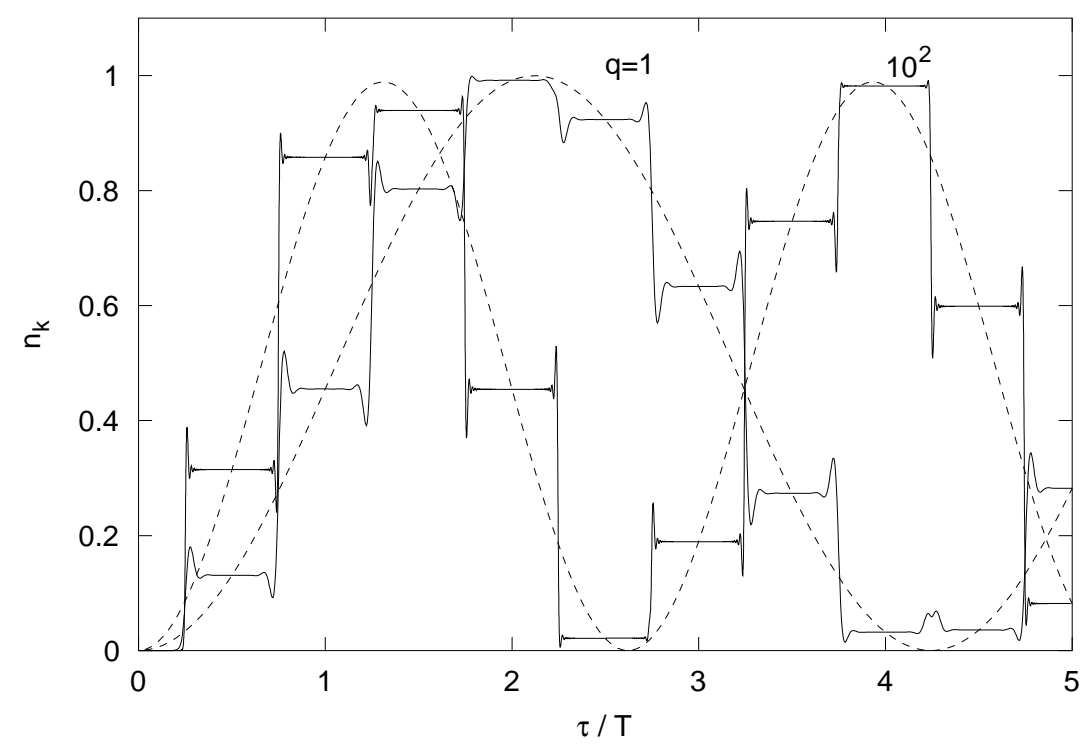

Figure 2: The time evolution of the fermion occupation number $n_{k}$ (continuous line) and the averaged particle number $\bar{n}_{k}$ (dashed line), for values of $k^{2}=0.51$ and 1.4 , corresponding to the first peaks of $F_{k}$ for $q_{1}=1$ and 100 respectively.

Note that $n_{k}(0)=0$, thanks to the initial conditions (3. easy to see that the occupation number of fermion pairs is always smaller than one, ${ }^{2}$ as expected.

Particle production through parametric resonance occurs at those moments when the adiabaticity condition $\mathrm{d} \Omega_{k} / \mathrm{d} \tau<\Omega_{k}^{2}$ is violated, see ref. [i] duction this will occur in general whenever the effective fermion mass $\bar{m}_{\psi}+\sqrt{q_{1}} y$ approaches zero. This is illustrated in figure ${ }_{2}^{2}$, where the continuous lines show the evolution of the exact occupation number for massless fermions and two values of the $q_{1}$ parameter. The occupation numbers $n_{k}$ (solid lines) have jumps every quarter and three quarters of the inflaton period, corresponding to the times when the inflaton value vanishes, $y=0$ (see figure $\left.\right|_{-1} ^{\prime} i_{1}$ ), and therefore when the effective fermion mass also vanishes.

To evaluate eq. (

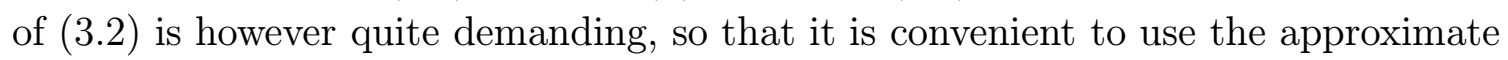

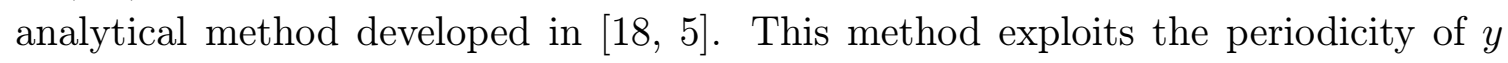
and allows to obtain a smooth function

$$
\bar{n}_{k}(\tau)=F_{k} \sin ^{2}\left(\nu_{k} \tau\right)
$$

which coincides with $n_{k}(\tau)$ for $\tau=n T$. Hence, $\bar{n}_{k}(\tau)$ gives an approximate expression for $n_{k}(\tau)$ without its fine (spiky) details. The approximate solution (dashed lines in

\footnotetext{
${ }^{2}$ This is related to the fact that $n_{k}=\left|\beta_{k}\right|^{2}=1-\left|\alpha_{k}\right|^{2} \leq 1$, for fermions, in terms of the Bogoliubov transformation coefficients $\left(\alpha_{k}, \beta_{k}\right)$, see ref. [1 $\left.1 \overline{8}^{\prime}\right]$.
} 


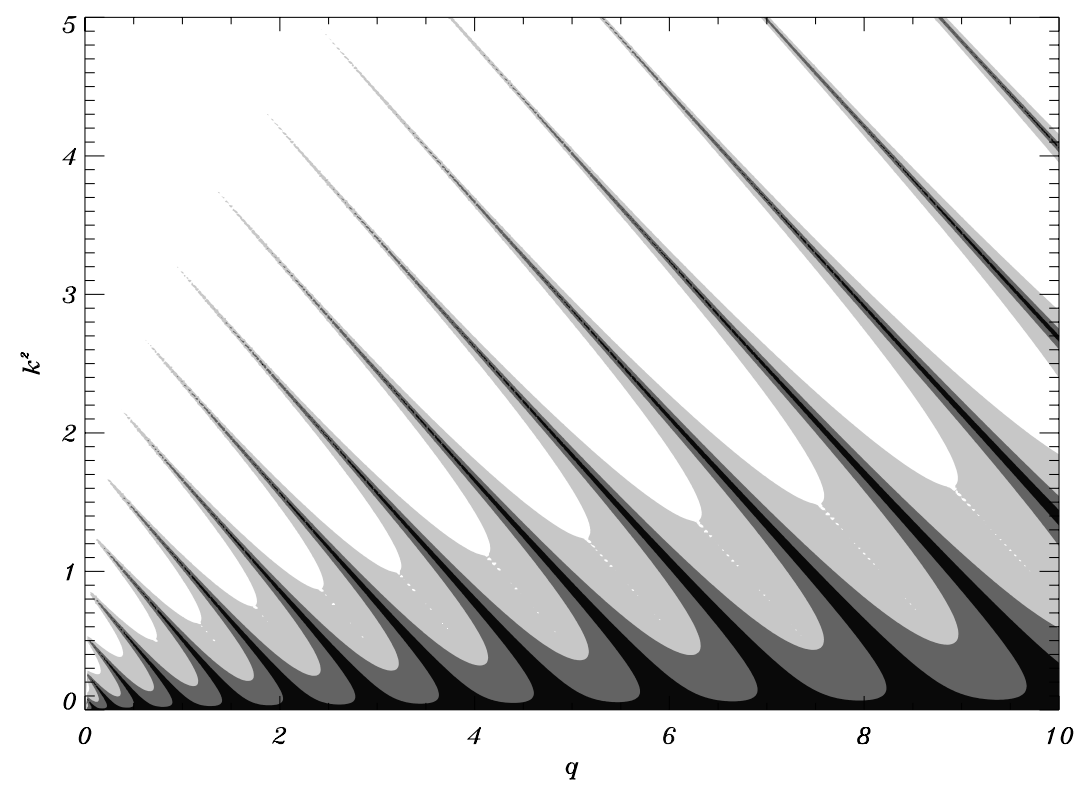

Figure 3: The instability chart for fermions coupled to the inflaton. Contours correspond to equipotential values of $F_{k}=0.1,0.5$ and 0.9 in the plane $\left(q_{1}, k^{2}\right)$ from lighter to darker.

figure ${ }^{1}$ ili follows the overall oscillations of the occupation number, matching the exact results at every inflaton period (and also at every half period in this case).

The advantage of using $\bar{n}_{k}(\tau)$ is that it has a simple temporal behavior, while its $k$ dependence can be obtained from the knowledge of the functions $F_{k}$ and $\nu_{k}$, where

$$
\begin{aligned}
F_{k} & =\frac{k^{2}\left(\operatorname{Im} X_{k}^{(1)}(T)\right)^{2}}{\Omega_{k}^{2}(T) \sin ^{2}\left(\nu_{k} T\right)}, \\
\cos \left(\nu_{k} T\right) & = \pm \operatorname{Re} X_{k}^{(1)}(T)
\end{aligned}
$$

and $X_{k}^{(1)}$ satisfies the same equation (13.2) with the initial condition $X_{k}^{(1)}(0)=1$, $X_{k}^{(1)^{\prime}}(0)=0$. Therefore, to obtain $\bar{n}_{k}(\tau)$ we only need to solve eq. (iㅡ. inflaton period. The two signs in eq. ( $\left.\underline{b}_{-} \overline{1}_{-} \overline{0}_{1}^{\prime}\right)$ correspond to two possible functions $\bar{n}_{k}$, which oscillate with the same amplitude but different frequency, and both match $n_{k}$ at every period of the inflaton oscillation. The best approximation to $n_{k}(\tau)$ is given by the one with smaller frequency $\nu_{k}$ for the $k^{2}$ values where $F_{k}$ is maximum (i.e. for the momenta contributing significantly to $\rho_{F}$ ), which in this case corresponds to

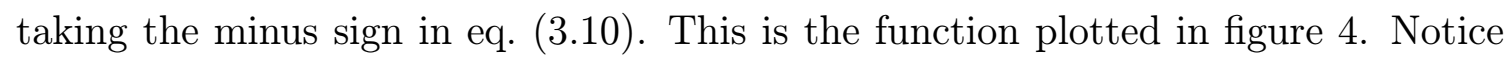
that the maximum value of $\nu_{k}$ is $\pi / T \simeq 0.105$, as can be deduced from eq. (3.1.

We show in figure $i_{-1}$ the instability chart for massless fermions coupled to the inflaton. It displays the contours in the $\left(q_{1}, k^{2}\right)$ plane of equal $F_{k}$ values. Fermions are mainly produced with momenta in the darker regions, corresponding to maxima of $F_{k}$. We see that the bands get narrower with increasing $k^{2}$ for a given $q_{1}$ value, and after several bands they shrink to a negligible width. 

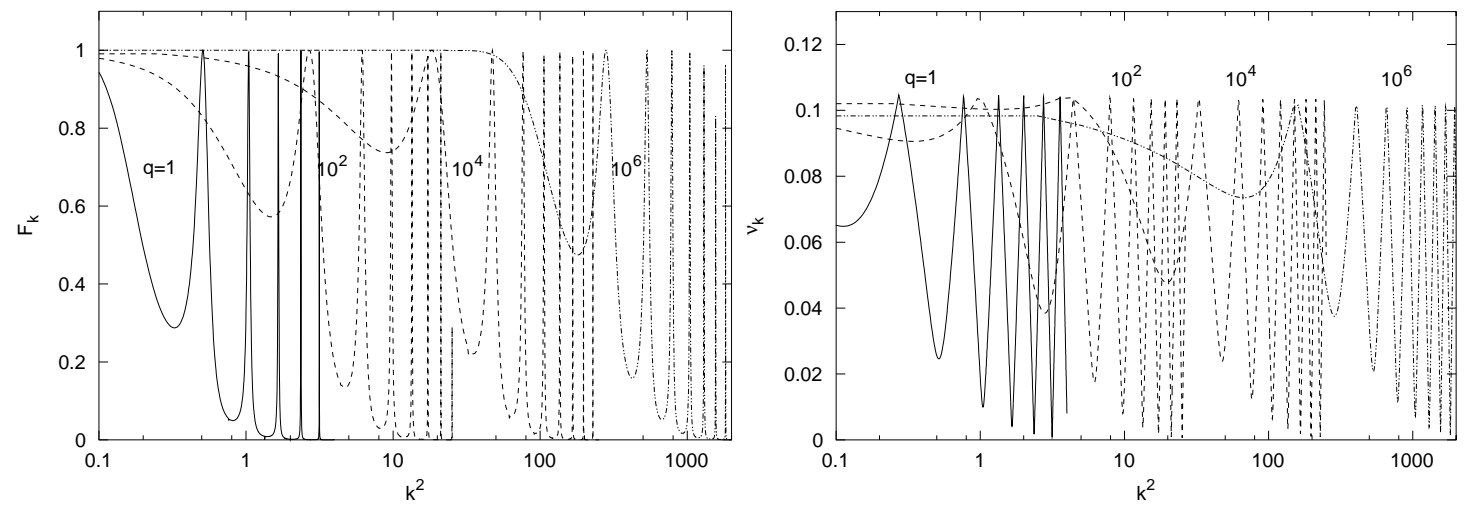

Figure 4: The fermion (envelope) spectrum $F_{k}$ (left panel) and $\nu_{k}$ (right panel) as a function of $k^{2}$, for fermions coupled to the inflaton, with $q_{1}=1,10^{2}, 10^{4}, 10^{6}$.

The left panel in figure ${ }_{-1}^{-1}$ shows the behavior of $F_{k}$ for different $q_{1}$ values extending up to $q_{1}=10^{6}$. These correspond to cuts in the instability chart (figure ${ }^{\prime} \overline{1}$ ) at a fixed value of $q_{1}$. The maximum momentum for which the bands are sizeable grows as $k_{\max } \sim q_{1}^{1 / 4}$ for fermions coupled to the inflaton. ${ }^{3}$ The right panel of figure $\bar{A}_{-1}$ shows $\nu_{k}$, as a function of $k^{2}$, for the same values of $q_{1}$. Note that the maxima of $F_{k}$ correspond to local minima of $\nu_{k}$.

The fraction of the total energy transferred to the fermions then finally results

$$
\frac{\rho_{F}(\tau)}{\rho_{\text {total }}}=h_{1}^{2} \frac{2 N}{\pi^{2} q_{1}} \int d k k^{2} \Omega_{k}(\tau) F_{k} \sin ^{2}\left(\nu_{k} \tau\right) .
$$

Its time evolution is shown in figure coupling parameter $q_{1}$. We see that after the first oscillation of the inflaton this fraction already reaches its asymptotic value and then fluctuates around it. The asymptotic value $\rho_{F} / \rho_{\text {total }}$ scales as $q_{1}^{1 / 4} h_{1}^{2}$. Moreover, for large values of $q_{1}$ a significant fraction of the inflaton energy can be transferred into fermions (as long as $h_{1}$ is not too small). We have checked that the final density is insensitive to the initial value of

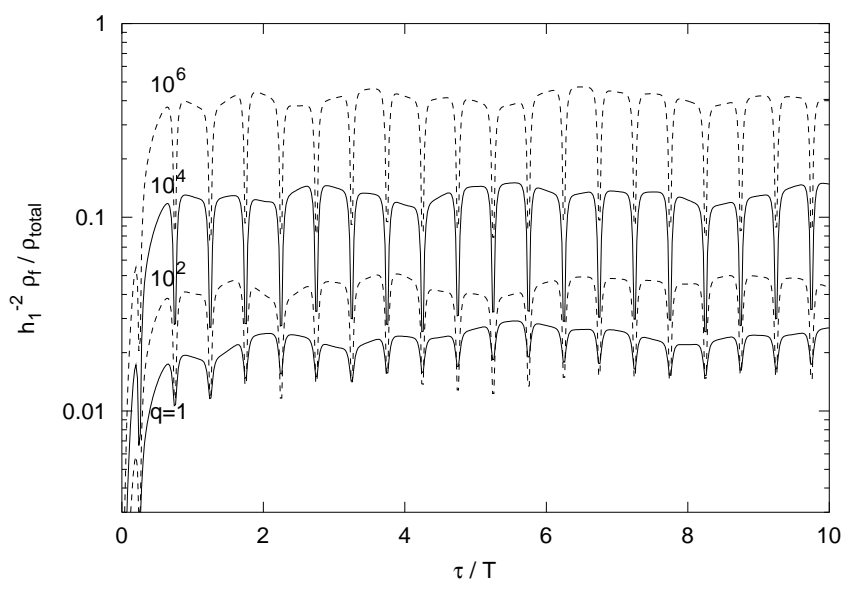

Figure 5: The fraction of the total energy transferred to fermions coupled to the inflaton, as a function of time, for $q_{1}=1,10^{2}, 10^{4}, 10^{6}$.

\footnotetext{
${ }^{3}$ Note that this is the same behavior as found in ref. $\left[\bar{p}^{-1}\right]$ for chaotic inflation. A different behavior, $k_{\max } \sim q^{1 / 3}$, was found in ref. $[\overline{7}]$ and was attributed, in their case, to the redshift of the modes during the particle production process.
} 


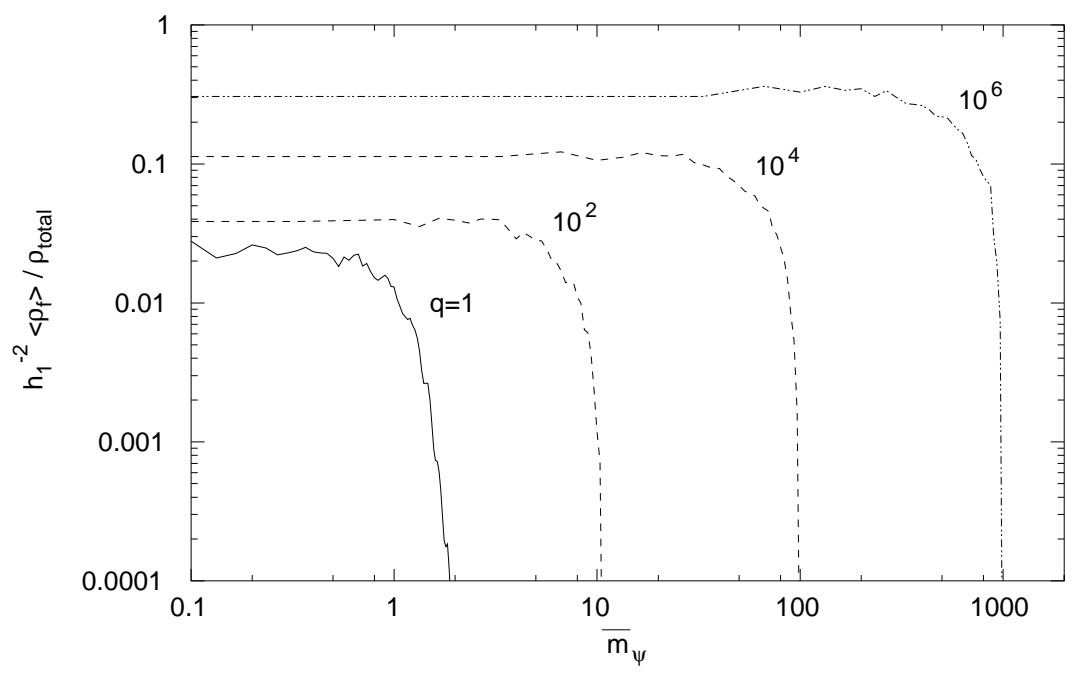

Figure 6: The fraction of the average energy density in fermions, $h_{1}^{-2}\left\langle\rho_{F}\right\rangle / \rho_{\text {total }}$ as a function of the fermion mass $\bar{m}_{\psi}$ (averaged between the eighth and tenth inflaton periods).

the inflaton field $y_{0}$ as long as $\left|y_{0}-1\right| \leq 10^{-2}$. This can be simply understood since, for $m_{\psi}=0$, the production of fermions takes place as the inflaton crosses through $y=0$.

If fermions are massive, the inflaton energy is transferred to the fermions with nearly the same efficiency as for massless fermions up to a maximum cut off value of the fermion's mass, above which it drastically drops. Figure ${ }_{-1}^{6} \overline{6}$ shows this behavior for different values of the $q_{1}$ parameter. The cut off value of the mass goes like ${ }^{4}$ $\bar{m}_{\psi, \text { cutoff }} \sim q_{1}^{1 / 2}$. The reason is simple, particle production occurs whenever the effective fermion mass vanishes, $m_{\text {eff }}=\bar{m}_{\psi}+\sqrt{q_{1}} y(\tau)=0$. For small values of $q_{1}$, even a small (positive) $\bar{m}_{\psi}$ will prevent $m_{\text {eff }}$ from vanishing as the inflaton $y(\tau)$ oscillates. However, as we increase $q_{1}$, larger bare masses are still allowed for particle production. The largest (negative) value of $y(\tau) \simeq-1$ gives the cutoff mass. Recalling that the actual mass of the fermion is $m_{\psi}=(g / \sqrt{\lambda}) \bar{m}_{\psi} M$, and that we are working on the regime $M<g M_{\mathrm{P}}$, we see that this still allows for quite large fermion masses to be produced.

\subsection{Coupling to the Higgs}

We will consider now the coupling of fermions to the Higgs, and study their pair production. The associated Mathieu equation is analogous to ( $\left(\underline{3} . \overline{2}_{1}^{\prime}\right)$, but with $x(\tau)=$ $+\sqrt{1-y(\tau)^{2}}$ in the place of $y(\tau)$, i.e.

$$
X_{k}^{\prime \prime}+\Omega_{k}^{2} X_{k}-i \sqrt{q_{2}} x^{\prime} X_{k}=0,
$$

where $\Omega_{k}^{2}=k^{2}+q_{2} x^{2}, \quad x^{\prime}=-y^{\prime} y / x$, and $q_{2}=h_{2}^{2} / g^{2}$.

\footnotetext{
${ }^{4}$ The same behaviour was found for chaotic inflation in ref. [i].
} 


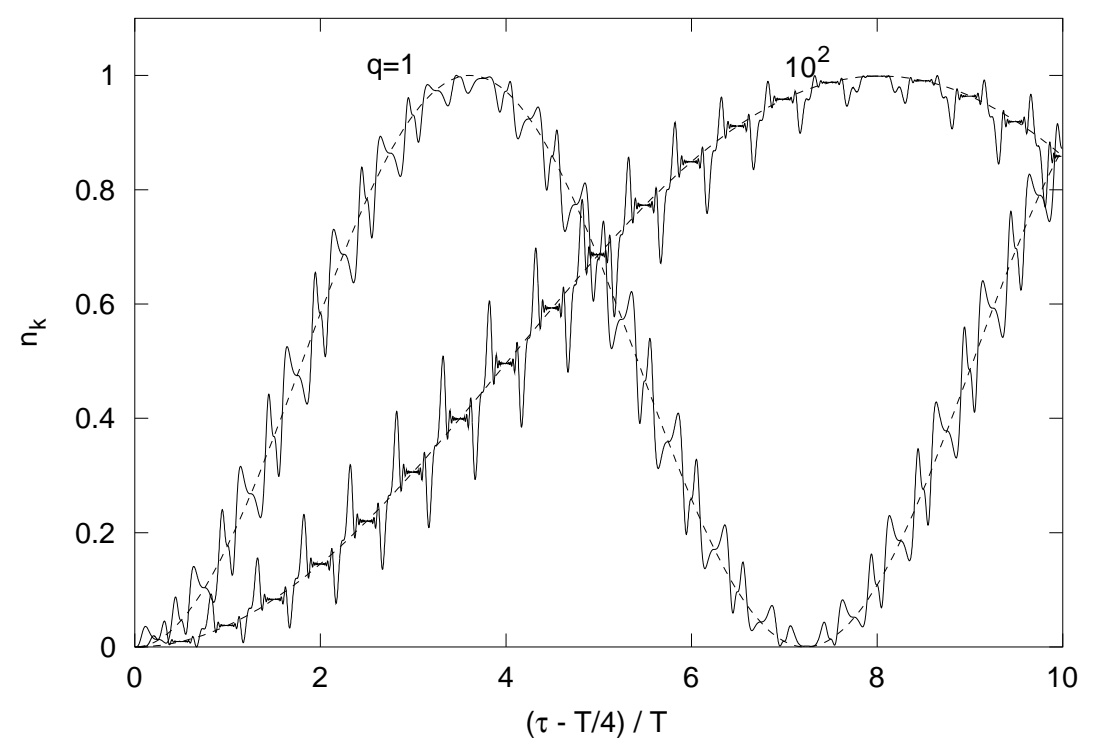

Figure 7: The time evolution of the fermion occupation number $n_{k}$ (continuous line) and the approximating function $\bar{n}_{k}$ (dashed line), for values of $k^{2}=0.235$ and 0.371 , corresponding to peaks of $F_{k}$ for $q_{2}=1$ and 100 respectively.

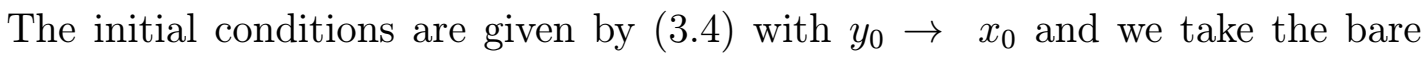
mass $\bar{m}_{\psi}=0$, since we are assuming that the fermion acquires a mass through the Higgs mechanism.

The occupation number is given by

$$
n_{k}(\tau)=\frac{1}{2}-\frac{k^{2}}{\Omega_{k}} \operatorname{Im}\left[X_{k} X_{k}^{\prime *}\right]-\frac{\sqrt{q_{2}} x}{2 \Omega_{k}} .
$$

Particle production again occurs periodically. In this case, the jumps in the occupation number take place for every period and half period of the inflaton, i.e. at $\tau=n T / 2$. These correspond to the times when the Higgs approaches zero, and thus when the effective mass of the fermion is at a minimum. An approximate expression $\bar{n}_{k}(\tau)$ can be constructed as in the previous case of fermions coupled to the inflaton. However, a function that matches $n_{k}(\tau)$ at the times when it has the spikes would not generally be a good approximation to the $n_{k}(\tau)$. Thus we have performed a shift of a quarter of period in the initial time, so that $\bar{n}_{k}(\tau)$ matches the value of $n_{k}(\tau)$ at $\tau=(2 n+1) T / 4$, i.e. where $n_{k}(\tau)$ has the plateau, and this gives a very good approximation as it is shown in figure $t_{-i}$ Starting with $n_{k}(\tau)=0$ at $\tau=T / 4$ does not affect the results after a few inflaton oscillations, since the density rapidly saturates to its asymptotic value.

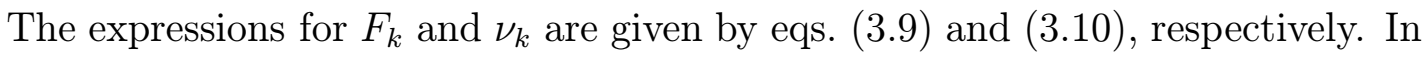
this case the function with smaller frequency in the peaks of $F_{k}$ corresponds to the solution of eq. $\left(\overline{3}_{3} . \overline{1} \overline{0}_{1}^{\prime}\right)$ with the plus sign choice. We show in figure chart for fermions coupled to the Higgs. The resonance bands are narrower than those corresponding to the coupling to the inflaton. 


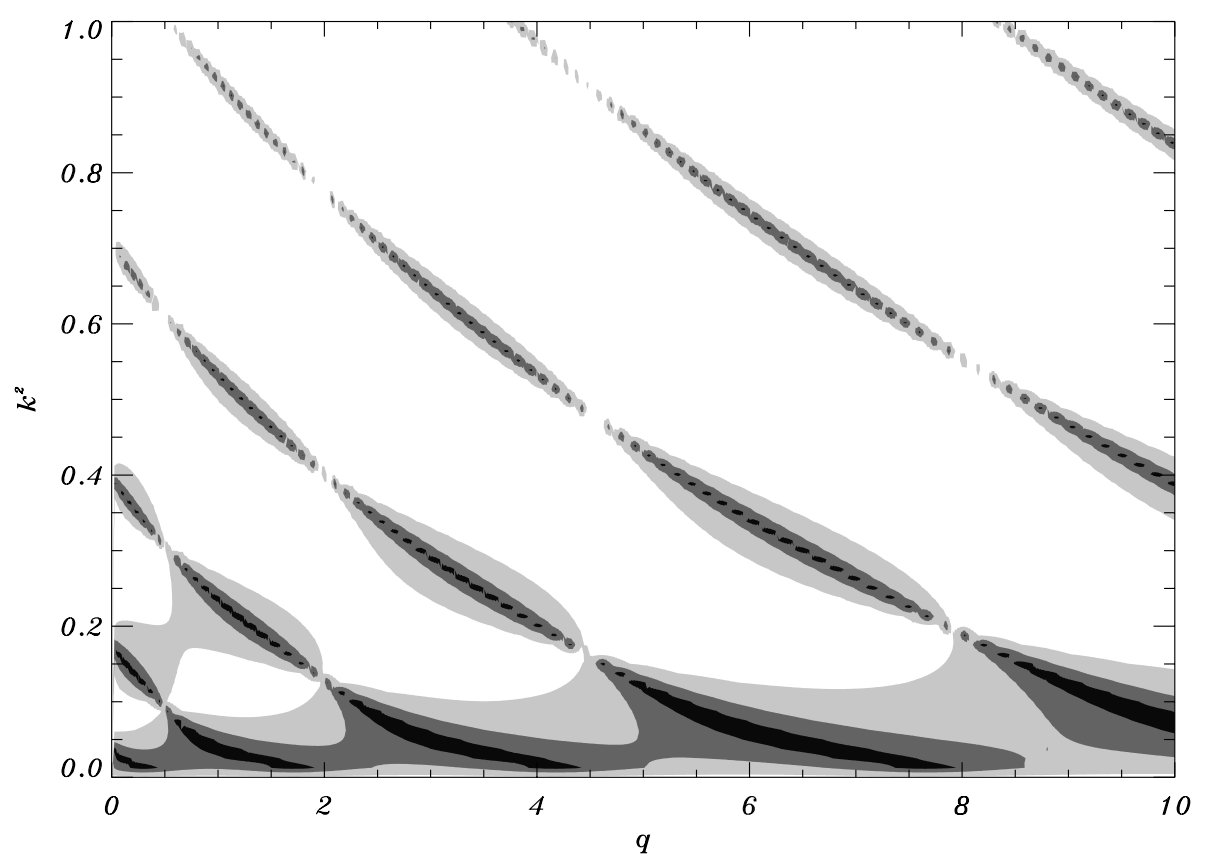

Figure 8: The instability chart for fermions coupled to the Higgs. Contours correspond to equipotential values of $F_{k}=0.1,0.5$ and 0.9 in the plane $\left(q_{2}, k^{2}\right)$.
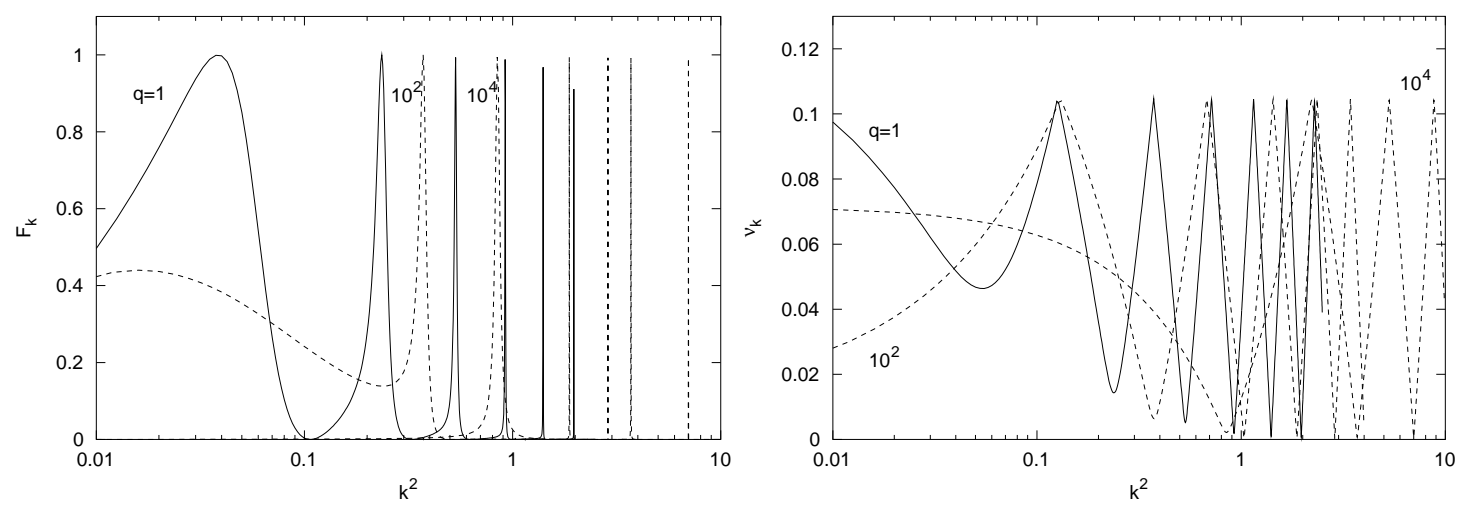

Figure 9: The fermion (envelope) spectrum $F_{k}$ (left panel) and $\nu_{k}$ (right panel) as a function of $k^{2}$, for fermions coupled to the Higgs, with $q_{2}=1,10^{2}, 10^{4}$. Note how narrow the peaks become for increasing values of $q_{2}$.

The left panel in figure ${ }_{-1}^{1}$ shows $F_{k}$ as a function of $k^{2}$ for several values of $q_{2}$, between 1 and $10^{4}$, while the right panel shows the corresponding $\nu_{k}$. Note how quickly the bands become very narrow as $k^{2}$ is increased. As a consequence, the fermion production through the coupling to the Higgs is in general less efficient than through the coupling to the inflaton in hybrid inflation models.

The ratio of Higgs-coupled fermion energy density to total energy is given by

$$
\frac{\rho_{F}(\tau)}{\rho_{\text {total }}}=\frac{g^{2} h_{2}^{2}}{\lambda} \frac{2 N}{\pi^{2} q_{2}} \int d k k^{2} \Omega_{k}(\tau) \bar{n}_{k}(\tau)
$$




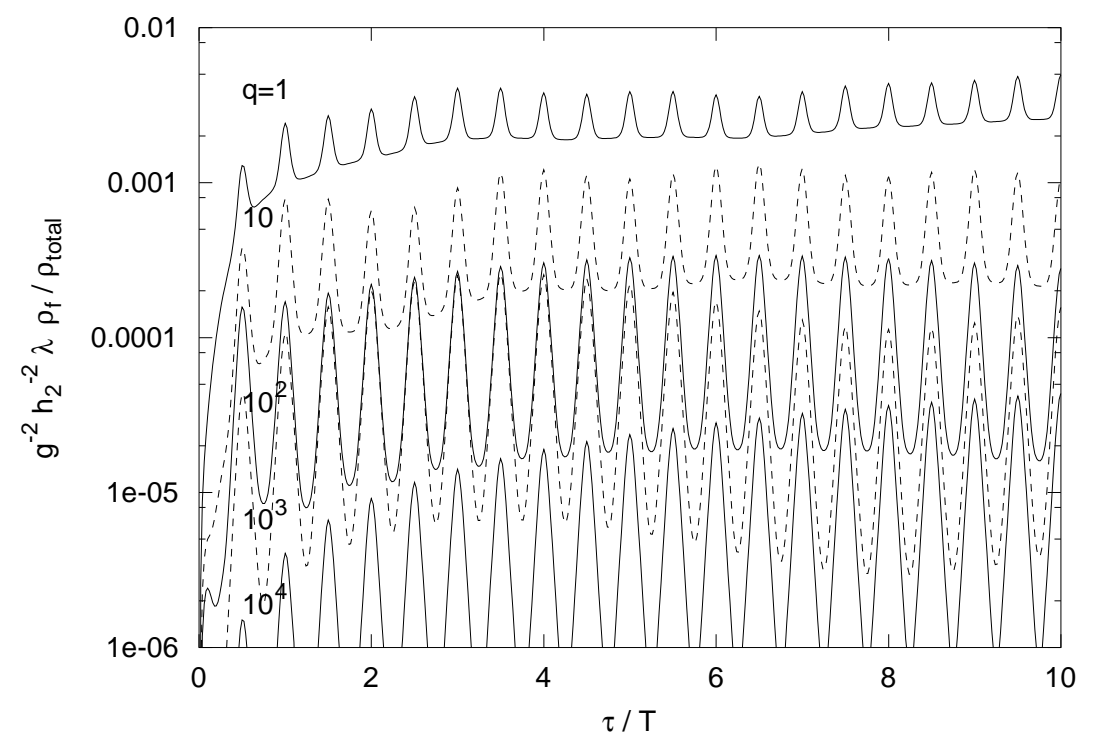

Figure 10: The fraction of the total energy transferred to fermions coupled to the Higgs, as a function of time, for $q_{2}=1,10,10^{2}, 10^{3}, 10^{4}$.

and it is shown in figure 'i 10 - If we take as a crude fit of the numerical results that $\left(\lambda / g^{2} h_{2}^{2}\right) \rho_{F} / \rho_{\text {total }} \sim 10^{-2} / q_{2}$, we see that $\rho_{F} / \rho_{\text {total }} \sim 10^{-2} g^{4} / \lambda$, and since we are working in the regime $\lambda \gg g^{2}$, we see that the fraction of energy transferred to fermions here remains small (but not necessarily negligible).

Since the fermionic production takes place when $x \simeq 0$, it is necessary to take the initial condition $y_{0}$ sufficiently close to one so that small Higgs values occur. Taking, as we did, $y_{0}=0.9999$, ensures that at least $x \sim 10^{-2}$ is reached, and hence the parametric resonance is not inhibited by a mass gap for the values of $q_{2}$ considered.

Notice that when the symmetry is finally broken, the fermions get a mass $m_{\psi}=$ $h_{2} M / \sqrt{\lambda}$, which also in this case can be quite large.

\section{Boson production}

The parametric resonant production of Higgs field particles has been shown to be quite inefficient in hybrid inflation models [3i]. However, other scalar particles coupled to the inflaton or the Higgs can can be abundantly produced. Here we will compute the parametric production of these bosons in order to compare it with the fermionic production.

We consider a scalar field $\chi$ with mass $m_{\chi}$ and coupling to $\phi$ and $\sigma$ given by

$$
V(\chi)=\frac{1}{2} m_{\chi}^{2} \chi^{2}+\frac{1}{2} g_{1}^{2} \chi^{2} \sigma^{2}+\frac{1}{2} g_{2}^{2} \chi^{2} \phi^{2} .
$$

We can write the equation of motion for the scalar field modes $X_{k}$ as

$$
X_{k}^{\prime \prime}+\Omega_{k}^{2}(\tau) X_{k}(\tau)=0,
$$


where

$$
\begin{aligned}
\Omega_{k}^{2}(\tau) & =k^{2}+\bar{m}_{\chi}^{2}+q_{1}^{\prime} y^{2}(\tau)+q_{2}^{\prime}\left(1-y^{2}(\tau)\right), \\
q_{1}^{\prime} & =\frac{\lambda g_{1}^{2}}{g^{4}}, \quad q_{2}^{\prime}=\frac{g_{2}^{2}}{g^{2}}, \quad \bar{m}_{\chi}=\frac{m_{\chi}}{\bar{M}} .
\end{aligned}
$$

We chose initial conditions of positive frequency plane waves at $\tau<0$

$$
\begin{aligned}
& X_{k}(0)=\left[2 \Omega_{k}\right]^{-1 / 2}, \\
& X_{k}^{\prime}(0)=-i \Omega_{k} X_{k}(0) .
\end{aligned}
$$

The boson occupation number can then be calculated as

$$
n_{k}(\tau)=\frac{1}{2 \Omega_{k}}\left|X_{k}^{\prime}\right|^{2}+\frac{\Omega_{k}}{2}\left|X_{k}\right|^{2}-\frac{1}{2} .
$$

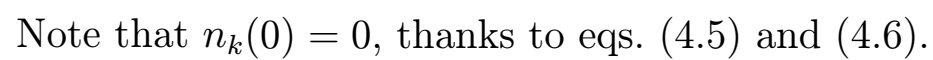

As in the case of the fermion production, we will use the method of ref. [1] 8 i] to compute an approximate expression for the boson occupation number

$$
\bar{n}_{k}(\tau)=2 \sinh ^{2}\left(\mu_{k} \tau\right)
$$

where the Floquet index or growth factor $\mu_{k}$ is determined by:

$$
\cosh \left(\mu_{k} T\right)=\operatorname{Re} X_{k}^{(1)}(T),
$$

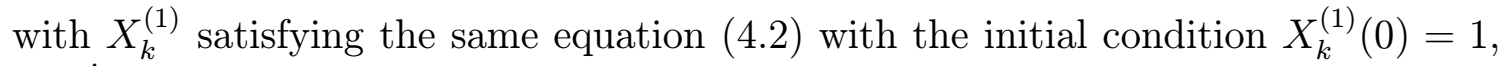
$X_{k}^{(1)^{\prime}}(0)=0$. The energy density of bosons can be obtained using this approximate solution as

$$
\rho_{B}(\tau)=\frac{1}{2 \pi^{2}} \int d k k^{2} \Omega_{k}(\tau) \bar{n}_{k}(\tau) .
$$

and therefore, the ratio of boson to total energies is

$$
\frac{\rho_{B}(\tau)}{\rho_{\text {total }}}=\frac{4 g_{1(2)}^{2}}{\pi^{2} q_{1(2)}} \int d k k^{2} \Omega_{k}(\tau) \sinh ^{2}\left(\mu_{k} \tau\right) .
$$

We show in figure per panel) and to the Higgs (lower panel). Shaded areas correspond to the instability bands for boson production. The darker areas correspond to larger Floquet index $\mu_{k}$, and thus to a more efficient particle production. In the unshaded areas there is no exponential boson production. The instability bands are thinner in the case of the coupling to the Higgs compared to those corresponding to the coupling to the inflaton, and they shrink to zero for specific values $q_{2}^{\prime}=1,3,6,10$, etc. This reflects the fact that modes with $q_{2}^{\prime}=n(n+1) / 2$ have no instabilities [i $\left.1 \overline{9}\right]$.

In figure $1 \overline{1} \overline{2}_{1}$ we show the Floquet index $\mu_{k}$ for bosons coupled to the inflaton and different values of the parameters $q_{1}^{\prime}$ up to $10^{6}$, corresponding to vertical slices in the 

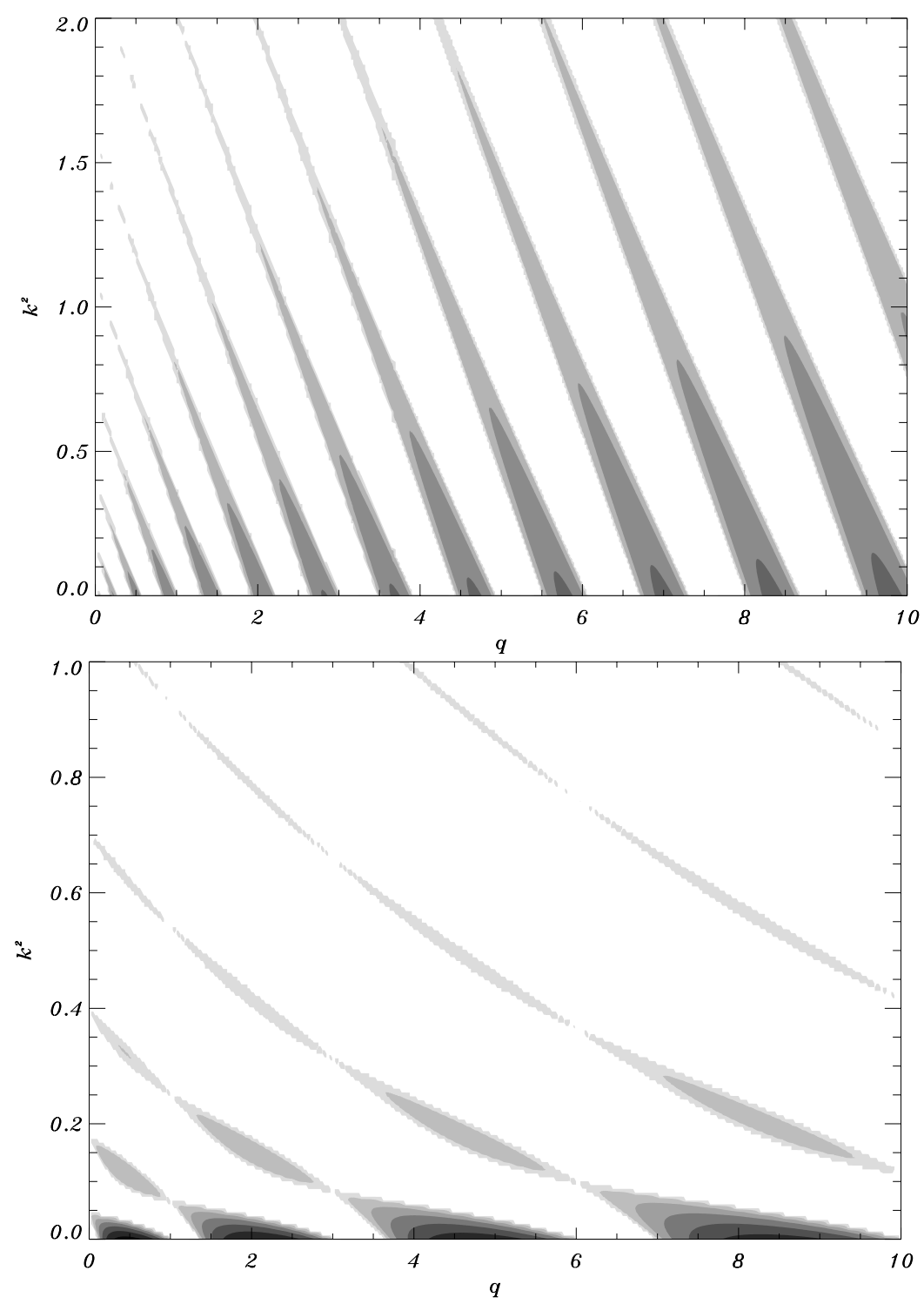

Figure 11: The instability chart for bosons coupled to the inflaton (upper panel) and to the Higgs (lower panel). Contours correspond to equipotential values of $\mu_{k}=10^{-3}, 0.01$, 0.03, 0.05, 0.07 and 0.09 in the plane $\left(q_{1(2)}^{\prime}, k^{2}\right)$.

instability charts of figure and extend up to larger $k^{2}$ with increasing $q_{1}^{\prime}$ values. Figure $1 \overline{1} \overline{3_{1}^{\prime}}$ shows the fraction of the total energy transferred to bosons coupled to the inflaton. Its mean value grows exponentially with time. The exponent is larger for larger $q_{1}^{\prime}$ values and the boson production is more efficient. When this fraction approaches unity, the backreaction effect becomes important and the results will be modified [6] be compared with those in figures ' Higgs respectively. It is clear that during the first few oscillations of the inflaton, the energy transfer to fermions can be more important than that to bosons. 


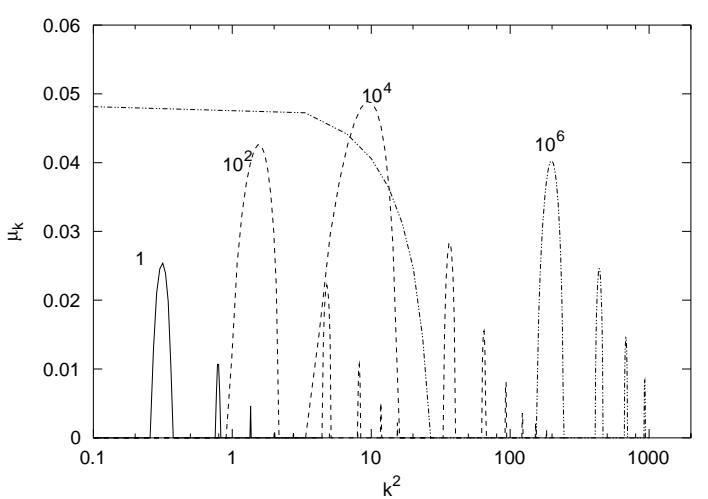

Figure 12: The boson growth index $\mu_{k}$ as a function of mode number $k^{2}$, for bosons coupled to the inflaton. The curves are labelled by the value of its $q_{1}^{\prime}$ parameter. Note that the bands become wider with larger $q_{1}^{\prime}$.

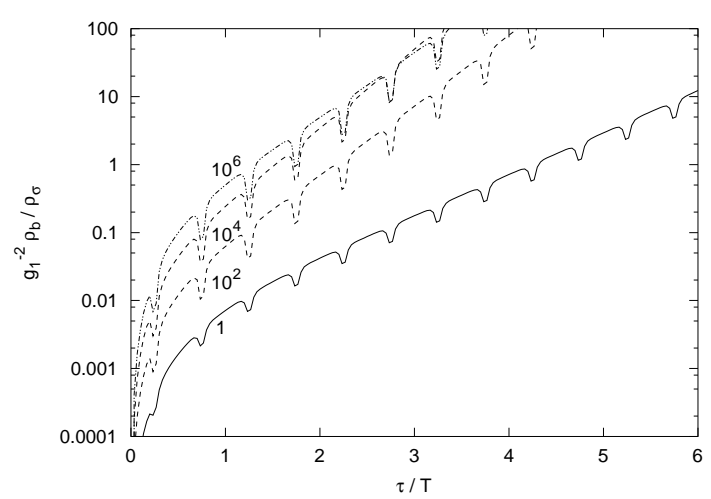

Figure 13: The fraction of the total energy transferred to bosons coupled to the inflaton, as a function of time, for different values of the $q_{1}^{\prime}=1,10^{2}, 10^{4}, 10^{6}$ (labelling the curves).

In the case that the bosons are coupled to the Higgs, as we noticed above, the particle production vanishes for $q_{2}^{\prime}=n(n+1) / 2$, and thus no energy is transferred to fermions for those $q_{2}^{\prime}$ values. We show in figure $1 \overline{1}$ the fraction of the total energy transferred to bosons as a function of time for a set of $q_{2}^{\prime}$ values spanning the range between the two zeroes, corresponding to $n=3$ and 4. The efficiency of bosonic production has an oscillatory behavior with increasing $q_{2}^{\prime}$ : it is maximal for $q_{2}^{\prime} \sim n^{2} / 2$ and minimal (zero) for

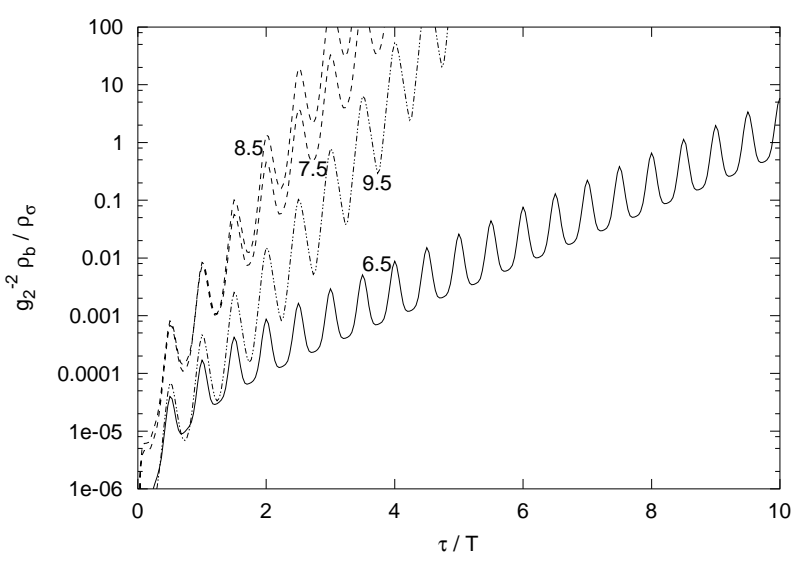

Figure 14: The fraction of the total energy transferred to bosons coupled to the Higgs as a function of time for different values of the $q_{2}^{\prime}=$ $6.5,7.5,8.5,9.5$ parameter (labelling the curves). $q_{2}^{\prime}=n(n+1) / 2$.

\section{Conclusions}

In this paper we have studied the parametric resonant production of fermions in hybrid inflation, with both fields, inflaton and Higgs coherently oscillating after inflation. We have assumed that fermions may couple to either the inflaton or the Higgs (or both). The behavior in the two cases is very different. While fermion production is very important in the case of a coupling to the inflaton, even in the presence of a bare mass, the production of fermions coupled only to the Higgs is 
generically weak. This is related to the fact that the non-adiabaticity condition, $d \Omega_{k} / d \tau>\Omega_{k}^{2}$, is harder to achieve for the Higgs since, when $\Omega_{k}$ is at a minimum, $d \Omega_{k} / d \tau$ is also at a minimum (contrary to the inflaton case). When the bare fermion mass exceeds the value $\bar{m}_{\psi}>\sqrt{q_{1}}$ the fermion production by the coupling to the inflaton field is also suppressed.

We have studied the growth of the fermion energy density and seen that it very quickly saturates to an approximately constant value. For fermions coupled to the inflaton, the asymptotic value grows with the resonance parameter like $h_{1}^{2} q_{1}^{1 / 4}$. For natural values of the couplings, a significant fraction of the inflaton energy can be transferred to fermions. On the other hand, for fermions coupled to the Higgs, the fermion energy density is of the order $\rho_{F} / \rho_{\text {total }} \sim 10^{-2} g^{4} / \lambda$, which under our working assumptions $\left(g^{2} \ll \lambda\right)$ is quite small.

We have also studied the boson production for both couplings to inflaton and Higgs, and compared with the fermion production. While the energy density transferred to the parametrically produced fermions saturates after a few oscillations, the one in bosons grows exponentially with time. Hence, if both fermions and bosons have similar couplings, most of the particle production goes initially into fermions, while at late times the boson production is exponentially dominant. There are however some values of boson couplings to the Higgs, corresponding to $q_{2}^{\prime}=n(n+1) / 2$, which completely inhibit the parametric resonance of bosons.

When the energy density of the bosonic or fermionic particles produced becomes sizeable, they are expected to backreact on the inflaton, affecting its evolution and eventually suppressing the parametric production of particles. We have not considered this process in detail in this paper since the approach followed is not the most suited one for this purpose. For a proper discussion of this issue in the context of chaotic inflation, see refs. [i], i i

As a summary, we have shown that the production of fermions in the preheating stage of hybrid inflation can be very important. For the range of model parameters assumed, hybrid inflation models lead to a more efficient fermion production than chaotic inflation models [i] the resonance parameter $q$. Depending on the relative size of the couplings, and on the backreaction process, the inflaton energy transferred to fermions may even be larger than that transferred to bosons. At any rate, the parametric production of out of equilibrium fermions could have interesting consequences for cosmological issues such as the generation of the baryon asymmetry through e.g. the leptogenesis mechanism.

Note added: while completing the writing of this work a related paper [20] appeared where the gravitino production during preheating is computed in a supersymmetric model of hybrid inflation. 


\section{Acknowledgments}

The work of J.G.B. has been supported in part by a CICYT project AEN/97/1678. S.M. and E.R. acknowledge financial support from CONICET, Fundación Antorchas and Agencia Nacional de Promoción Científica y Tecnológica. S.M. and E.R. thank Centro Atómico Bariloche for hospitality during the completion of this work.

\section{References}

[1] L. Kofman, A. Linde and A.A. Starobinsky, Reheating after inflation, 'Phys. Rev. Lett.' ( Phys. Rev. D 56 (1997) 3258

[2] P.B. Greene, L. Kofman, A. Linde and A.A. Starobinsky, Structure of resonance in

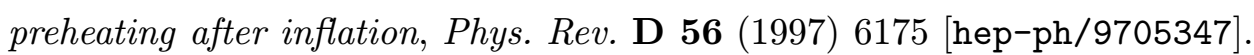

[3] J. García-Bellido and A. Linde, Preheating in hybrid inflation, Phys. Rev. D 57_(1998) -

[4] P.B. Greene, L. Kofman and A.A. Starobinsky, Sine-Gordon parametric resonance,

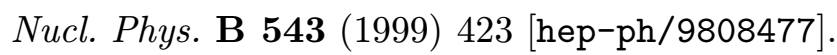

[5] P.B. Greene and L. Kofman, Preheating of fermions, 'P

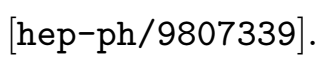

[6] J. Baacke, K. Heitmann and C. Patzold, Nonequilibrium dynamics of fermions in a spatially homogeneous scalar background field, 'Phys. Rev. hep-ph/9806205i].

[7] G.F. Giudice, M. Peloso, A. Riotto and I. Tkachev, Production of massive fermions at

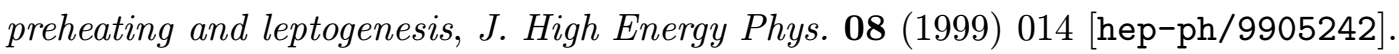

[8] E.W. Kolb, A. Linde and A. Riotto, Gut baryogenesis after preheating, îhys. $\bar{R} \bar{e} \bar{v}$.

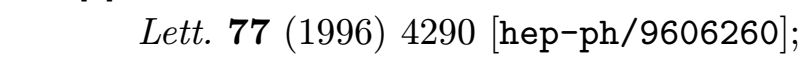

G.W. Anderson, A. Linde and A. Riotto, Preheating, supersymmetry breaking and baryogenesis, 'Phys. Rev. Lett. 77 (1996) 3716 [hep-ph/9606416'.

[9] J. García-Bellido, D.Y. Grigoriev, A. Kusenko and M. Shaposhnikov, Non-equilibrium

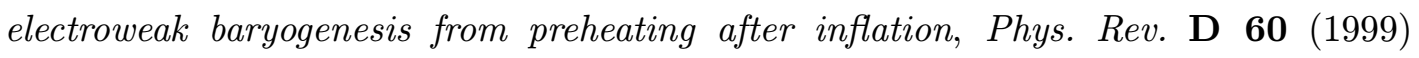
;

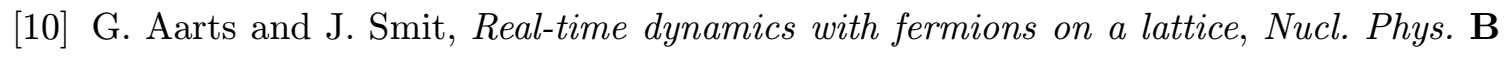

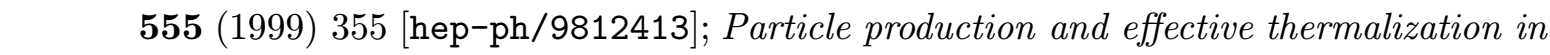
inhomogeneous mean field theory, 'P

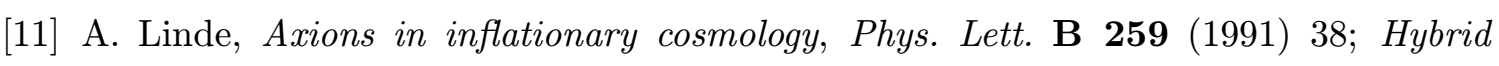

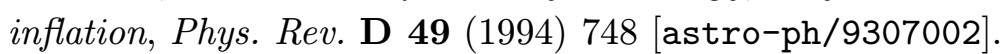


[12] J. García-Bellido and D. Wands, The spectrum of curvature perturbations from hybrid inflation, 'Phys. Rev. D 54 (1996) 7181' [astro-ph/9606047'.

[13] E.J. Copeland, A.R. Liddle, D.H. Lyth, E.D. Stewart and D. Wands, False vacuum inflation with Einstein gravity, 'Phys. Rev. D 49 (1994) 6410 [astro-ph/9401011].

[14] J. García-Bellido, A.D. Linde and D. Wands, Density perturbations and black hole

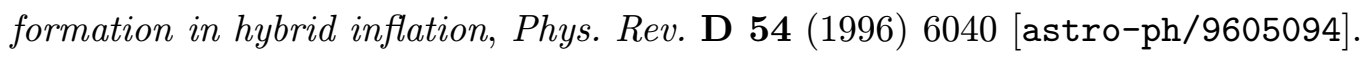

[15] For a recent review, see D.H. Lyth and A. Riotto, Particle physics models of inflation and the cosmological density perturbation, 'Phys. Rep. 314 (1999) 1, [hep-ph/9807278].

[16] D.H. Lyth, The parameter space for tree-level hybrid inflation, hep-ph/9904371.

[17] M. Bastero-Gil, S.F. King and J. Sanderson, Preheating in supersymmetric hybrid

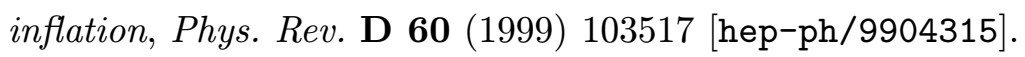

[18] A.A. Grib, S.G. Mamaev and V.M. Mostepanenko, Quantum effects in strong external fields, Friedmann Laboratories Pub. Co., San Petersburg 1994.

[19] J. García-Bellido, in preparation.

[20] M. Bastero-Gil and A. Mazumdar, Gravitino production in hybrid inflationary models, hep-ph/0002004 